\title{
A note for the Ulam-Hyers-Rassias stability of differential equations on bounded intervals
}

\author{
Serkan Asliyüce and Süleyman Ö̆̆rekçi \\ Amasya University, Department of Mathematics, Amasya, Turkey \\ Received: 30 December 2020, Accepted: 30 June 2021 \\ Published online: 12 September 2021.
}

Abstract: In this paper, we considered the stability problem of non-linear differential equations in the sense of Ulam-Hyers and UlamHyers-Rassias on bounded intervals. We show that some widely used restrictions commonly assumed in similar problems are not necessary. Therefore we extend and improve some well-known results by dropping some of their assumptions.

Keywords: Differential equations; Stability theory; Ulam-Hyers-Rassias Stability; Fixed point theory; Generalized metric spaces.

\section{Introduction}

In 1940, S.M. Ulam [1] posed the following problem in a talk given at Wisconsin University: "Under what conditions does there exists a homomorphism near an approximately homomorphism of a complete metric group?, i.e. Given a metric group $(\mathrm{G}, \mathrm{d})$, a number $\varepsilon>0$ and a mapping $f: G \rightarrow G$ satisfying the inequality

$$
d(f(t u), f(t) f(u))<\varepsilon
$$

for all $t, u \in G$, does there exist a homomorphism $g$ of $G$ and a constant $K$, depending only on $G$, such that

$$
d(f(t), g(t))<K \varepsilon
$$

for all $t, u \in G$ ?" And if there is such homomorphism, then the equation $g(t u)=g(t) g(u)$ of the homomorphism is called stable.

In 1941, Hyers [2] answered this problem: Let $B_{1}$ and $B_{2}$ be real Banach spaces and $\varepsilon>0$. Then for each $f: B_{1} \rightarrow B_{2}$ satisfying the inequality

$$
\|f(t+u)-f(t)-f(u)\| \leq \varepsilon
$$

for all $t, u \in B_{1}$, there exists a unique $g: B_{1} \rightarrow B_{2}$ such that

$$
\|f(t)-g(t)\| \leq \varepsilon
$$

holds for all $t \in B_{1}$.

In 1978, Rassias [3] introduced a remarkable generalization of this problem considering the constant $\varepsilon$ as a variable function, this type problems known as Ulam-Hyers-Rassias stability problems in the literature. 
First paper on the Ulam-Hyers type stability of the differential equations is given by Obloza [4, ?]. After that Alsina and Ger [6] proved that every differentiable mapping $y: I \rightarrow \mathbb{R}$, where $I$ is an open interval of real numbers, satisfying $\left|y^{\prime}(t)-y(t)\right| \leq \varepsilon$ for all $t \in I$ and a given $\varepsilon>0$, there exists a solution $t_{0}$ of the differential equation $y^{\prime}(t)=y(t)$ such that $\left|y(t)-y_{0}(t)\right| \leq 3 \varepsilon$ for all $t \in I$. This result was later extended by Takahasi et. al. to the equation $y(t)=\lambda y(t)$ in Banach spaces, and to higher-order linear differential equations with constant coefficients, see $[7, ?, ?]$. Following these pioneering works, many authors studied this subject, see $[10,11,12,13,14]$ and references therein.

In 2010, S.M. Jung [10] proved Ulam-Hyers and Ulam-Hyers-Rassias stability of the equation

$$
y^{\prime}(t)=f(t, y)
$$

using the fixed point technique. This result is important since it extends the previous results to the non-linear case. Bojor [15] modified this technique to investigate the stability of the linear equation

$$
y^{\prime}(t)+f(t) y(t)=g(t)
$$

The fixed point technique proposed by Jung is used widely used to analyze the Ulam-Hyers and Ulam-Hyers-Rassias stability of the differential equations in different types.

Başcı et.al. [11] investigated the Ulam-Hyers and Ulam-Hyers-Rassias stability of the equation

$$
y^{\prime}(t)=f(t, y)
$$

using Jung's method with a modification on the metric. Contrary to Jung, they used the generalized metric defined as

$$
d(f, g):=\inf \left\{C \in[0, \infty]:|f(t)-g(t)| e^{-M\left(t-t_{0}\right)} \leq C \Phi(t), t \in I\right\}
$$

Here, we will show the condition

$$
\left|\int_{t_{0}}^{t} \varphi(s) d s\right| \leq K \varphi(t)
$$

used by Başcı et.al. to prove the Ulam-Hyers-Rassias stability of the (1) is unnecessary, and then Ulam-Hyers-Rassias stability implies Ulam-Hyers stability the on the bounded intervals.

This paper is organized as follows: In Section 2, we gave the definitions of Ulam-Hyers stability, Ulam-Hyers-Rassias stability and generalized metric. And, we stated the fixed point theorem that will be used in the sequel. In Section 3 , we stated and proved our main result and noted some remarks about it. We devoted last section to the evaluation of the article.

\section{Preliminaries}

Let $I$ be an open interval. For every $\varepsilon \geq 0$ and $y \in \mathscr{C}^{1}(I)$ satisfying

$$
|y(t)-f(t, y(t))| \leq \varepsilon
$$

() 2021 BISKA Bilisim Technology 
if there exists a solution $y_{0}$ of the Eq. (1) such that

$$
\left|y(t)-y_{0}(r)\right| \leq K \varepsilon
$$

where $K$ is a constant which does not depend on $\varepsilon$ and $y$, then the differential equation (1) is said to be stable in the sense of Ulam-Hyers. If the above statement remains true after replacing the constants $\varepsilon$ and $K$ with the functions $\phi, \Phi: I \rightarrow[0, \infty)$ respectively, where these functions do not depend on $y$ and $y_{0}$, then the differential equation (1) is said to be stable in the sense of Ulam-Hyers-Rassias. This definition may be applied to different classes of differential equations, we refer to Jung [10] and references cited therein for more detailed definitions of Ulam-Hyers stability and Ulam-Hyers-Rassias stability.

Now we introduce the generalized metric which will be used in the sequel. For a non-empty set $X$, a function $d: X \times X \rightarrow$ $[0, \infty]$ is called a generalized metric on $X$ if and only if satisfies,

$\mathrm{M}(1) d(t, u)=0$ if and only if $t=u$,

$\mathrm{M}(2) d(t, u)=d(u, t)$ for all $t, u \in X$,

$\mathrm{M}(3) d(t, w) \leq d(t, u)+d(u, w)$ for all $t, u, w \in X$.

We will use the following fixed point theorem in the proof of our main theorem.

Theorem 1. [16] Let $(X, d)$ is a generalized complete metric space. Assume that $T: X \rightarrow X$ is a strictly contractive operator with the Lipschitz constant $L<1$. If there is a non-negative integer $m$ such that $d\left(T^{m+1} x, T^{m} x\right)<\infty$ for some $x \in X$, then the following are true:

(a) The sequence $\left\{T^{m} x\right\}$ converges to a fixed point $\bar{x}$ of $T$,

(b) $\bar{x}$ is the unique fixed point of $T$ in

$$
\bar{X}=\left\{y \in X: d\left(T^{m} x, y\right)<\infty\right\}
$$

(c) If $y \in \bar{X}$, then

$$
d(y, \bar{x}) \leq \frac{1}{1-L} d(T y, y)
$$

\section{Main results}

In this section, we define the interval $I$ as $I:=\left[t_{0}, t_{0}+r\right], t_{0}, r \in \mathbb{R}$ and $r>0$. And we define the set of all continuous functions defined on $I$ by

$$
X:=\{f: I \rightarrow \mathbb{R} ; f \text { is continuous }\}=\mathscr{C}(I, \mathbb{R}) .
$$

Lemma 1. $(X, d)$ is a generalized complete metric space with the metric

$$
d(f, g):=\inf \left\{c \in[0, \infty] ;|f(t)-g(t)| e^{M\left(t-t_{0}\right)} \leq c \Phi(t), t \in I\right\}
$$

where $M>0$ is a constant and $\Phi: I \rightarrow(0, \infty)$ is a continuous.

For the proof of this lemma, we refer to [11].

Our next result is about the Ulam-Hyers-Rassias stability of the Eq. (1).

Theorem 2. Let $f: I \times \mathbb{R} \rightarrow \mathbb{R}$ be a continuous function satisfying the Lipschitz condition

$$
|f(t, g)-f(t, h)| \leq L|g-h|
$$


for all $t \in I$ and all $g, h \in \mathbb{R}$. For a non-decreasing continuous function $\varphi: I \rightarrow(0, \infty)$ if a continuously differentiable function $y: I \rightarrow \mathbb{R}$ satisfies

$$
\left|y^{\prime}(t)-f(t, y(t))\right| \leq \varphi(t)
$$

for all $t \in I$, then there exists a unique solution $y_{0}$ of the Eq. (1) satisfying

$$
\left|y(t)-y_{0}(t)\right| \leq r(1+L) \varphi(t)
$$

for all $t \in I$.

Proof.Let the set $X$ be defined by (2). Define the $d: X \times X \rightarrow[0, \infty]$ by

$$
d(f, g):=\inf \left\{c \in[0, \infty] ;|f(t)-g(t)| e^{-(\alpha+1)\left(t-t_{0}\right)} \leq c \varphi(t), t \in I\right\} .
$$

Given Lemma 1 , one can conclude that $(X, d)$ is a generalized complete metric space. Define the operator $\mathscr{R}: X \rightarrow X$ by

$$
\mathscr{R} y(t)=y\left(t_{0}\right)+\int_{t_{0}}^{t} f(\tau, y(\tau)) d \tau,
$$

$t \in I$ and $y \in X$. It is clear that fixed points of $\mathscr{R}$ are solutions of Eq. (1). Since functions $f$ and $y$ are continuous functions, we can say that $\mathscr{R} y$ is continuous and it belongs to the set $X$. Hence, it is easy to see $d\left(\mathscr{R} h_{0}, h_{0}\right)<\infty$ for all $h_{0} \in X$ and $\left\{h \in X ; d\left(h_{0}, h\right)<\infty\right\}=X$.

For any $h_{1}, h_{2} \in X$, let $c_{h_{1}, h_{2}} \in[0, \infty]$ be a constant such that $d\left(h_{1}, h_{2}\right) \leq c_{h_{1}, h_{2}}$, i.e.

$$
\left|h_{1}(t)-h_{2}(t)\right| e^{-(\alpha+1)\left(t-t_{0}\right)} \leq c_{h_{1}, h_{2}} \varphi(t), t \in I .
$$

Now, we can show that operator $\mathscr{R}$ is strictly contractive. For any $h_{1}, h_{2} \in X$ and all $t \in I$, using (3), (4), and monotonicity of $\varphi$, we obtain that

$$
\begin{aligned}
\left|\mathscr{R} h_{1}(t)-\mathscr{R} h_{2}(t)\right| & =\left|\int_{t_{0}}^{t}\left[f\left(\tau, h_{1}(\tau)\right)-f\left(\tau, h_{2}(\tau)\right)\right] d \tau\right| \\
& \leq \int_{t_{0}}^{t}\left|f\left(\tau, h_{1}(\tau)\right)-f\left(\tau, h_{2}(\tau)\right)\right| d \tau \\
& \leq L \int_{t_{0}}^{t}\left|h_{1}(\tau)-h_{2}(\tau)\right| \\
& =L \int_{t_{0}}^{t}\left|h_{1}(\tau)-h_{2}(\tau)\right| e^{-(\alpha+1)\left(\tau-t_{0}\right)} e^{(\alpha+1)\left(\tau-t_{0}\right)} d \tau \\
& \leq L c_{h_{1}, h_{2}} \int_{t_{0}}^{t} \varphi(\tau) e^{(\alpha+1)\left(\tau-t_{0}\right)} d \tau \\
& \leq L c_{h_{1}, h_{2}} \varphi(t) \int_{t_{0}}^{t} e^{(\alpha+1)\left(\tau-t_{0}\right)} d \tau \\
& \leq \frac{L}{L+1} c_{h_{1}, h_{2}} \varphi(t) e^{(\alpha+1)\left(t-t_{0}\right)} .
\end{aligned}
$$

(๑) 2021 BISKA Bilisim Technology 
Therefore, we have

$$
\left|\mathscr{R} h_{1}(t)-\mathscr{R} h_{2}(t)\right| e^{-(\alpha+1)\left(t-t_{0}\right)} \leq \frac{L}{L+1} c_{h_{1}, h_{2}} \varphi(t),
$$

that is

$$
d\left(\mathscr{R} h_{1}, \mathscr{R} h_{2}\right) \leq \frac{L}{L+1} d\left(h_{1}, h_{2}\right) .
$$

So, operator $\mathscr{R}$ is strictly contractive on $X$, and all conditions of Theorem 1 are satisfied for $m=1$.

From (5), we have

$$
-\varphi(t) \leq y^{\prime}(t)-f(t, y(t)) \leq \varphi(t)
$$

for all $t \in I$. Integrating this inequality from $t_{0}$ to $t$ and using the monotonicity of the function $\varphi$, we obtain

$$
\left|y^{\prime}(t)-\mathscr{R} y(t)\right| \leq \int_{t_{0}}^{t} \varphi(\tau) d \tau \leq \varphi(t) \int_{t_{0}}^{t} d \tau \leq \varphi(t) r
$$

for all $t \in I$. Multiplying this last inequality with $e^{-(\alpha+1)\left(t-t_{0}\right)}$, we get

$$
\left|y^{\prime}(t)-\mathscr{R} y(t)\right| e^{-(\alpha+1)\left(t-t_{0}\right)} \leq \varphi(t) r e^{-(\alpha+1)\left(t-t_{0}\right)}
$$

for all $t \in I$, which implies

$$
d(\mathscr{R} y, y) \leq \varphi(t) r e^{-(\alpha+1)\left(t-t_{0}\right)}
$$

for all $t \in I$.

According to Theorem 1, there exists a unique solution $y_{0}: I \rightarrow \mathbb{R}$ of Eq. (1) satisfying

$$
d\left(y, y_{0}\right) \leq \frac{1}{1-L /(L+1)} d(\mathscr{R} y, y) \leq \frac{\varphi(t) r e^{-(\alpha+1)\left(t-t_{0}\right)}}{1-L /(L+1)}=r(1+L) \varphi(t) e^{-(\alpha+1)\left(t-t_{0}\right)}
$$

for all $t \in I$. Hence, from 3 , we obtain

$$
\left|y(t)-y_{0}(t)\right| e^{-(\alpha+1)\left(t-t_{0}\right)} \leq r(1+L) \varphi(t) e^{-(\alpha+1)\left(t-t_{0}\right)}
$$

for all $t \in I$. This inequality implies (6) and completes the proof.

Remark. Notice that we do not need the assumption

$$
\left|\int_{t_{0}}^{t} \varphi(s) d s\right| \leq K \varphi(t)
$$

in the proof of Theorem 2, which is required in [11]. This condition is required also in many papers concerning similar problems on bounded intervals, so we improve the literature with this result.

By taking $\varphi(t)=\varepsilon$ in Theorem 2, we obtain the following result on Ulam-Hyers stability of (1).

Corollary 1.Let $f: I \times \mathbb{R} \rightarrow \mathbb{R}$ be a continuous function satisfying the Lipschitz condition (4) for all $t \in I$ and all $g, h \in \mathbb{R}$. If a continuously differentiable function $y: I \rightarrow \mathbb{R}$ satisfies

$$
\left|y^{\prime}(t)-f(t, y(t))\right| \leq \varepsilon
$$


for all $t \in I$, then there exists a unique solution $y_{0}$ of the Eq. (1) satisfying

$$
\left|y(t)-y_{0}(t)\right| \leq r(1+L) \varepsilon
$$

for all $t \in I$.

\section{Conclusion}

In this study, we have considered the stability problem of the non-linear differential equation (1) in the sense of UlamHyers and Ulam-Hyers-Rassias on bounded intervals. With Theorem 2, we proved the Ulam-Hyers-Rassias stability of (1) with fewer assumptions than the analogue result stated in [11]. Also we have obtained Ulam-Hyers stability of the differential equation (1) as a corollary of Theorem 2.

The results we obtained here with fewer conditions can be applied to many different equations that previous methods cannot be used.

\section{Competing interests}

The authors declare that they have no competing interests.

\section{Authors' contributions}

All authors have contributed to all parts of the article. All authors read and approved the final manuscript.

\section{References}

[1] Ulam, S.M.: A Collection of Mathematical Problems. Interscience, Woburn (1960)

[2] Hyers, D.H.: On the stability of the linear functional equation. Proc. Nat. Acad. Sci. U.S.A. 27, $222-224$ (1941)

[3] Rassias, T.: On the stability of linear mappings in Banach spaces. Proc. Am.Math. Soc. 72, 297-300 (1978)

[4] Obloza, M.: Hyers-Ulam stability of the linear differential equations. Rocznik. Nauk. Dydakt. Prace. Mat. 13, $259-270$ (1993)

[5] Obloza, M.: Connections between Hyers and Lyapunov stability of the ordinary differential equations. Rocznik. Nauk. Dydakt. Prace. Mat. 14, 141-146 (1997)

[6] Alsina, C., Ger, R.: On some inequalities and stability results related to the exponential function. J. Inequal. Appl. 2, 373-380 (1998)

[7] Takahasi, S.H., Miura, T., Miyajima, S.: The Hyers-Ulam stability constants of first order linear differential operators. Bull. Korean Math. Soc. 39, 309-315 (2002)

[8] Miura, T., Miyajima, S., Takahasi, S.H.: A characterization of Hyers-Ulam stability of first order linear differential operators. J. Math. Anal. Appl. 286, 136-146 (2003)

[9] Miura, T., Miyajima, S., Takahasi, S.H.: Hyers-Ulam stability of linear differential operator with constant coefficients. Math. Nachr. 258, 90-96 (2003)

[10] Jung, S.M.: A fixed point approach to the stability of differential equations $y^{\prime}=f(x, y)$. Bull. Malays. Math. Sci. Soc. 33(1), 47-56 (2010)

[11] Başcı, Y., Mısır, A., Öğrekçi, S.: On the stability problem of differentail equations in the sense of Ulam. Results Math. 75:6, 13 pp. (2020)

[12] S. Ögrekçi. Stability of delay differential equations in the sense of Ulam on unbounded intervals. An International Journal of Optimization and Control: Theories \& Applications (IJOCTA), 9(2):125-131, 2019.

๑) 2021 BISKA Bilisim Technology 
[13] C. Tunç and E. Biçer. Hyers-Ulam-Rassias stability for a first order functional differential equation. J. Math. Fund. Sci., 47(2):143153, 2015.

[14] Y. Başcı, S. Öğrekçi, and A. Mısır. Hyers-Ulam-Rassias stability for Abel-Riccati type first-order differential equations. GU. J. Sci., 32(4):1238-1252, 2019.

[15] Bojor, F.: Note on the stability of first order linear differential equations. Opusc. Math. 32, 67-74 (2012)

[16] Diaz, J.B., Margolis, B.: A fixed point theorem of alternative, for contractions on a generalized complete metric space. Bull. Am. Math. Soc. 74, 305-309 (2003) 